Supporting Information for

\title{
Bubble-free transfer technique for high-quality graphene/hexagonal boron nitride van der Waals heterostructures
}

\author{
Takuya Iwasaki ${ }^{*}$, Kosuke Endo ${ }^{2,3}$, Eiichiro Watanabe ${ }^{4}$, Daiju Tsuya ${ }^{4}$, Yoshifumi Morita ${ }^{5}$, Shu \\ Nakaharai $^{2}$, Yutaka Noguchi ${ }^{3}$, Yutaka Wakayama ${ }^{2}$, Kenji Watanabe ${ }^{6}$, Takashi Taniguchi ${ }^{6}$, and \\ Satoshi Moriyama* \\ 1 International Center for Young Scientists (ICYS), National Institute for Materials Science \\ (NIMS), Tsukuba, Ibaraki 305-0044, Japan \\ ${ }^{2}$ International Center for Materials Nanoarchitectonics (WPI-MANA), NIMS, Tsukuba, Ibaraki \\ 305-0044, Japan \\ ${ }^{3}$ Department of Science \& Technology, Meiji University, Kawasaki 214-8571, Japan \\ ${ }^{4}$ Nanofabrication Platform, NIMS, Tsukuba, Ibaraki 305-0047, Japan \\ ${ }^{5}$ Faculty of Engineering, Gunma University, Kiryu, Gunma 376-8515, Japan \\ ${ }^{6}$ Research Center for Functional Materials, NIMS, Tsukuba, Ibaraki 305-0044, Japan \\ E-mail: IWASAKI.Takuya@nims.go.jp (T.I.), MORIYAMA.Satoshi@nims.go.jp (S.M.)
}




\section{Movie(1) for drop-down process in bubble-free transfer}

Movie(1) shows the captured optical image during the drop down process of hexagonal boron nitride (hBN) onto graphene. This process is described in Section 3-1 and Figures $2 a$ (vii),b(vii) in the main text. The speed of movie is multiplied by 5 . Yellow lines in the movie exhibit markers for alignment between hBN and graphene flakes. The stamp contact area in the left side gradually expanded to the right side by lifting up the substrate stage, until graphene flake was covered by the hBN flake. During this process, the objective lens 20x was used for minimizing unintentional misalignment.

\section{Movie(2) for peeling off process}

Movie(2) shows the captured optical image during the peeling off process of a thick graphite flake. This process is described in Section 3-2 and Figure 3 in the main text. The speed of movie is multiplied by 2. After contacting the center of the stamp, the stamp was moved to the left side. Finally, the stamp was retracted with the graphite flake. During this process, the objective lens $5 \mathrm{x}$ was used.

\section{Bubble formation}

As mentioned in Section 3-1 in the main text, the contact angle between the flakes is important to avoid the bubble formation. If the hBN is picked up by the center of the stamp (top of the hemispherical-shaped surface), the contact angle would be very small $\sim 0^{\circ}$. In this case, a lot of bubbles can be formed between flakes as shown in Figure S1. 

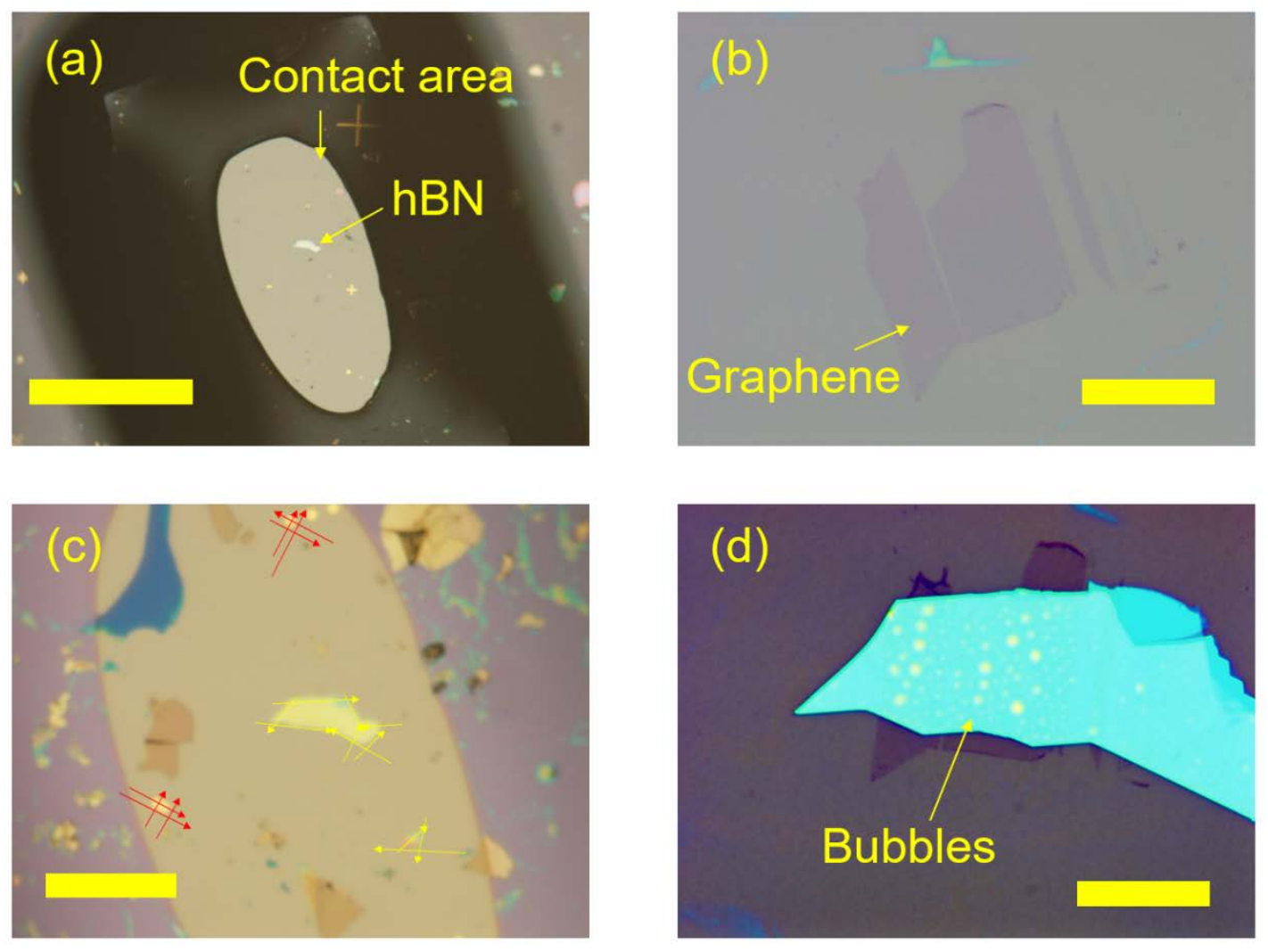

Figure S1. Optical images of transfer process of hBN picked-up at the center of the stamp. (a) Pick-up process of the hBN flake at the center of the contact area. (b) Optical image of the graphene flake on the $\mathrm{SiO}_{2} / \mathrm{Si}$ substrate. (c) Drop-down process of the hBN onto the graphene flake. Yellow and red lines show the markers for alignment. (d) hBN/graphene heterostructure after the transfer process. Many bubbles are obviously visible. Scale bars correspond to $500 \mu \mathrm{m}$ for (a), $20 \mu \mathrm{m}$ for (b), (d), $100 \mu \mathrm{m}$ for (c), respectively. 


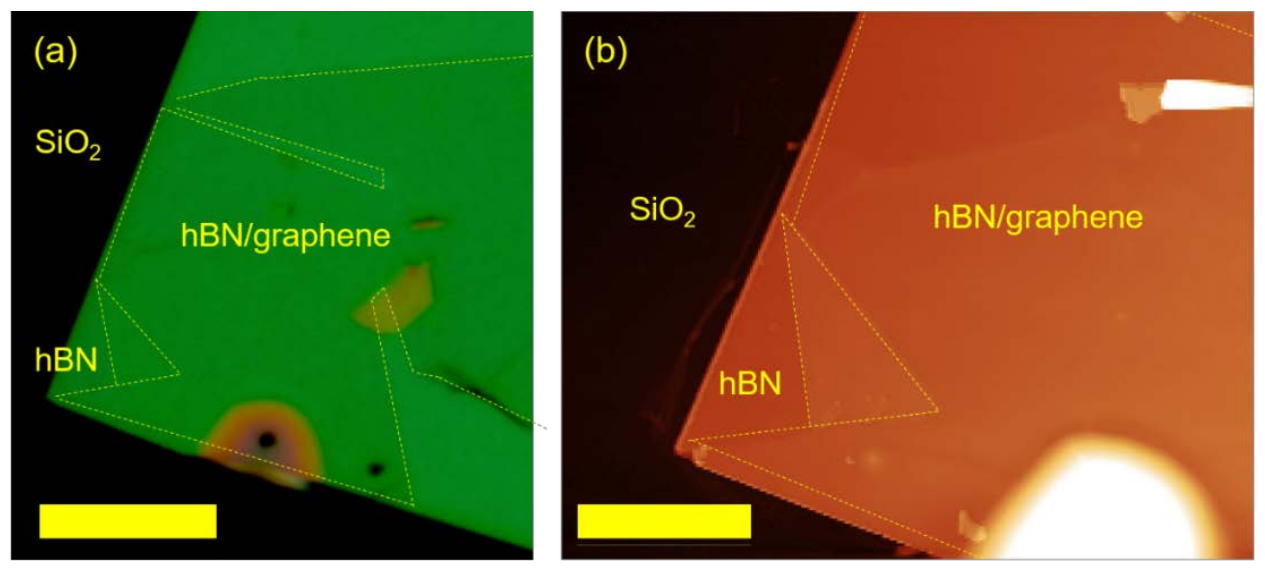

Figure S2. Large bubble free region in a hBN/graphene heterostructure. (a) Optical image. Scale bar corresponds to $10 \mu \mathrm{m}$. (b) AFM topography. Scale bar corresponds to $5 \mu \mathrm{m}$. Dashed yellow lines depict the edge of the graphene flake. 